\title{
Index of Major Topics
}

Note: all references to the body of the text are italicised and are according to the pagination of the editio princeps of Socrates from 1651, marked by square brackets throughout the present edition. All other references are given afterwards in regular Arabic numerals.

Alchemists 22

Anatomy 49, 98, 110, 131-32, 268-69

Anima mundi (World-soul) 132, 180, 183-84

Antiperistasis 267, 282

Aristotle's philosophy

Act and potency 141

Definition 276

Doctrine of the four causes 56

Ekthesis 223

First uncaused cause 173

Form, matter, and privation 22, 296

Principle of non-contradiction 157

Relatives, category of $17,31-33,43-44$, $112,162-63,229$

Substance and accidents $41-42,44,47$, $85-88,210,212$

Syllogism 215-19

Theory of natural place 130

Asininity 156

Astronomy 95, 174, 232, 261-64, 272, 28

Atomism 19, 81-83, 147

Authority (ille dixit) 8

Botany 70,213, 269

Chance $20-21,48,58,63,81-82,129,167-68$, $209,239,253-54$

Chess 23

Daedalus's statues 185

Divine mind 21, 254

Doctrine of animal spirits 138,164

Dreams 18, 37, 40, 45-46, 51-54, 59-60, 67, $83,120-22,135,146,178,206,294$

Eleatic school

Parmenides's concept of being 60

Zeno's paradoxes 235

Empedocles's theory of knowledge 110

Fortune $13,17,20,80-81,123,126,197,237-38$ $240,248,270,293$
Genius 49, 63, 65, 87, 100, 112-13, 153, 169, 196, 198, 225, 164, 309, 311-12, 492, 494

Gordian knot 106, 509

Happy medium 205, 249, 251, 510

Homo homini Deus 167, 507

Homo homini homo 167, 507

Homo homini lupus 167, 507

Homogeneous essences of things (Anaxagoras) 21,491

Humours (Hippocratic theory) 48, 212

Hydrographic maps 285

Infinite worlds 283

Know thyself (nosce te ipsum) 301

Law

Human 31

Mosaic: Dedication

Of Nature 38

Lethe, river of oblivion 180

Magnet 71-72, 76, 130, 133, 166, 304, 497

Man is the measure of all things (Protagoras) 14

Mathematics and geometry

Continuum 92, 224

Discrete numbers (integers) 60, 224

Doubling of the cube (Delian problem) 30

Euclidean geometry $49,59,68,73,78,119$, $162,210,213,223-24,226$

Irrational numbers 191, 233-34

Squaring the circle 92

Medusa 18

Mirrors 14, 26, 73-75, 78, 97, 116, 146, 247

Necessity 21, 26, 67, 82, 257-58

Nothingness 34

Originating principle of Nature

Air (Anaximenes) 18

Earth (Orpheus) 19 
Fire (Heraclitus) 18

Water (Thales) 18

Four elements (Empedocles) 19, 186-87

Physics

Centre of gravity 26

Platonic philosophy

Doctrine of recollection 128,185

Theory of Ideas 21, 185

Problem of universals 11-13, 142, 151-57, 228

Providence 259, 273, 277

Pythagorean philosophy $20,64,133,157,161$, 210,220

Ring of Gyges 290

Runaway slave 183

Scepticism

Apathy 14

Dove's neck 101

Equipollence 200

Infinite regress 125, 208, 224

Probable (pithanon) 4, 9, 13, 16, 22, 70, 88, $100,134,139,150,194,223,236,239$, $248,280,310,311$

Resembling the truth (verisimile) 58, 311

Sceptic 2, 43

Self-scrutiny $23,63,113$

Suspension of judgment (epochē) 4, 13-14, 16, 277, 307, 309, 315

Tranquility (ataraxia) $13-14,79-80$

Withholding of assent $11,13,277,307-8$, 312

Senses (external)

Hearing $31,33,42,64,73,76,98-99$, $125-26$

Sight $33,40,42,46-47,49-51,64-65,70$, $73,76,78,89,93,102,116,125-26$, $134,243,255$

Smell 42, 64, 70, 76, 125-26

Taste 42, 64, 73-74, 76, 125-26, 170

Touch 40-51, 64-65, 72, 76, 89-94, 125-26, 145, 193
Senses (internal)

Common sense $3,106,108-15$

Estimative 106

Imagination 3, 54, 91, 106,108, 114-16, 118-22, 124-25, 155, 158, 167, 182

Memory 3, 106, 123, 125-31, 181-82

Sophists $34-63,103-4,112,194,196,215,218$, 252, 255

Stoic philosophy $132,137-38,173,198-202$

Superstition $274,287-88,313$

Telescope (Galileo) 94

Theatrum mundi 19, 194, 276

Laughing philosopher (Democritus ridens)

18-19, 230, 272

Weeping philosopher (Heraclitus flens) 18

Time 149-51

True religion 274,313

Veritas filia temporis 242

Virtues

Beneficence 289-91

Friendship 297-99

Glory 294

Humility 299

Justice 292-93

Perseverance 294

Prudence $10,12,237-73$

Strength 283

Temperance 296

Vision, theory of $50-51,88,94,96-104,145-46$

Colours 48, 73, 87, 101-3, 113-14, 155, 159, $163,169,175-76,199,210$

Diffraction, phenomenon of 101

Emission of rays $66-70$

Simulacra 14, 65-69

Vices

Ambition 299-300

Avarice 299

Cruelty 300-301

Discord 300-301

Infidelity 301

Envy 300

Pride 299

Wooden dove of Archytas $85-86$ 
